\title{
Early onset of drinking increases alcohol use in adulthood
}

Pitkanen T, Lyyra AL, Pulkkinen L. Age of onset of drinking and the use of alcohol in adulthood: a follow-up study from age 8-42 for females and males. Addiction 2005; 100:652-61.

Does the age of onset of drinking affect adult alcohol use?

\section{METHODS}

-

Design: Longitudinal cohort study

Setting: Twelve classes of second grade pupils in Jyväskylä, Finland from 1968-2001. Population: 369 children in the second grade of school at study
enrolment.

Prognostic factors: Age of onset of drinking (assessed by personal interview alone at ages 14 and 20 , and by personal interview and Life Situation Questionnaire at ages 27,36, and 42)

If Outcomes: Adult alcohol use (frequency of drinking; frequency of binge drinking (defined as more than five drinks; scale from $0=$ never to $5=$ several times a week).

Follow up period: Thirty four years.

Age of onset of drinking and drinking habits at age 36-42 years

\begin{tabular}{|c|c|c|c|}
\hline & \multicolumn{2}{|c|}{ Onset age of drinking } & \multirow[b]{2}{*}{ p Value ${ }^{*}$} \\
\hline & $\leqslant 13$ years old & $\geqslant 18$ years old & \\
\hline \multicolumn{4}{|c|}{ Mean drinking frequency } \\
\hline Men & 193 days/year & 101.6 days/year & 0.005 \\
\hline Women & 110 days/year & 47.6 days/year & 0.011 \\
\hline \multicolumn{4}{|c|}{$\begin{array}{l}\text { Mean binge drinking } \\
\text { score }\end{array}$} \\
\hline Men & 3.2 & 1.8 & 0.000 \\
\hline Women & 1.9 & 0.7 & 0.004 \\
\hline
\end{tabular}

* $p$ values shown to three decimal points only.

For correspondence: Tuuli Pitkänen, Department of Psychology, University of Jyväskylä, PO Box 35 (Agora), FIN-40041, Jyväskylä, Finland; Tuuli.pitkanen@nic.fi

Source of funding: Academy of Finland (Finnish Centre of Excellence Programme).
MAIN RESULTS

Mean age of onset of drinking was 15 years 6 months old (range 10-30 years). People who started drinking at age 13 or younger had significantly increased frequency of alcohol use and binge drinking in adulthood than those who started drinking at age 18 or later $(\mathrm{p}<0.05$ for all outcomes, see table).

\section{CONCLUSIONS}

Adult alcohol use increases in people who start drinking at an early age. Interventions that delay the onset of drinking are likely to prevent alcohol problems in later life.

\section{Commentary}

his paper from the Finnish Jyväskylä Longitudinal Study of Personality and Social Development examines the age of onset of drinking as a predictor for heavy drinking in adulthood (measured as frequency of alcohol consumption and binge drinking, using two instruments for screening for alcoholism, the CAGE and modified Michigan Alcoholism Screening Test (Mm-MAST)). The study followed a sample of 369 people from the age of 8 years to the age of 36 years, and had a high response rate (over $90 \%$ ). Age of onset of drinking was found to be inversely related to adult alcohol use, with participants who started drinking at age 13 years or younger scoring more highly on all indicators of adult alcohol use than those who started at older ages. As pointed out in the paper, the findings of this study support results from previous longitudinal studies. The strength of this particular project, the authors argue, was that the age of onset of alcohol use was obtained by prospective measurement.

In terms of individual practice, the findings suggest the need for early intervention to reduce harmful alcohol consumption in individuals with an early onset of drinking. This may be in the form of brief intervention, psychotherapy, or pharmacotherapy for alcohol dependence, depending on the individual. Encouraging individuals at risk of long term alcohol problems to engage in treatment is a challenge and an area needing further exploration.

More broadly, these findings have important implications for public policy. The authors conclude that efforts to prevent alcohol related harm should include keeping "adulthood" as the legal age limit for alcohol use. This is very difficult to implement in many cultures today because of significant influences on the uptake of alcohol by young people, including widespread availability and acceptability of recurrent binge drinking as well as sophisticated promotion of alcohol to young people. Notwithstanding this, clinicians can and should advocate for appropriate alcohol related public policy, in particular highlighting the harms associated with early onset of alcohol and the need for responsible serving practices.

Yvonne Bonomo FRACP PhD FAChAM Department of Addiction Medicine, St Vincent's Health and Department of Paediatrics, University of Melbourne, Melbourne, Australia 